\title{
Geophysical Imaging of Stimulated Microbial Biomineralization
}

Kenneth H. Williams, Dimitrios Ntarlagiannis, Lee D. Slater, Alice Dohnalkova, Susan S. Hubbard, and Jillian F. Banfield

\section{Supporting Information}

The following supporting information consists of four pages containing four figures with captions. The figures are included to supplement the material presented in the manuscript. They include photographs to assist in the visualization of the column design and layout, as well as additional time-varying complex resistivity data supporting the hypothesis of mineral coarsening.

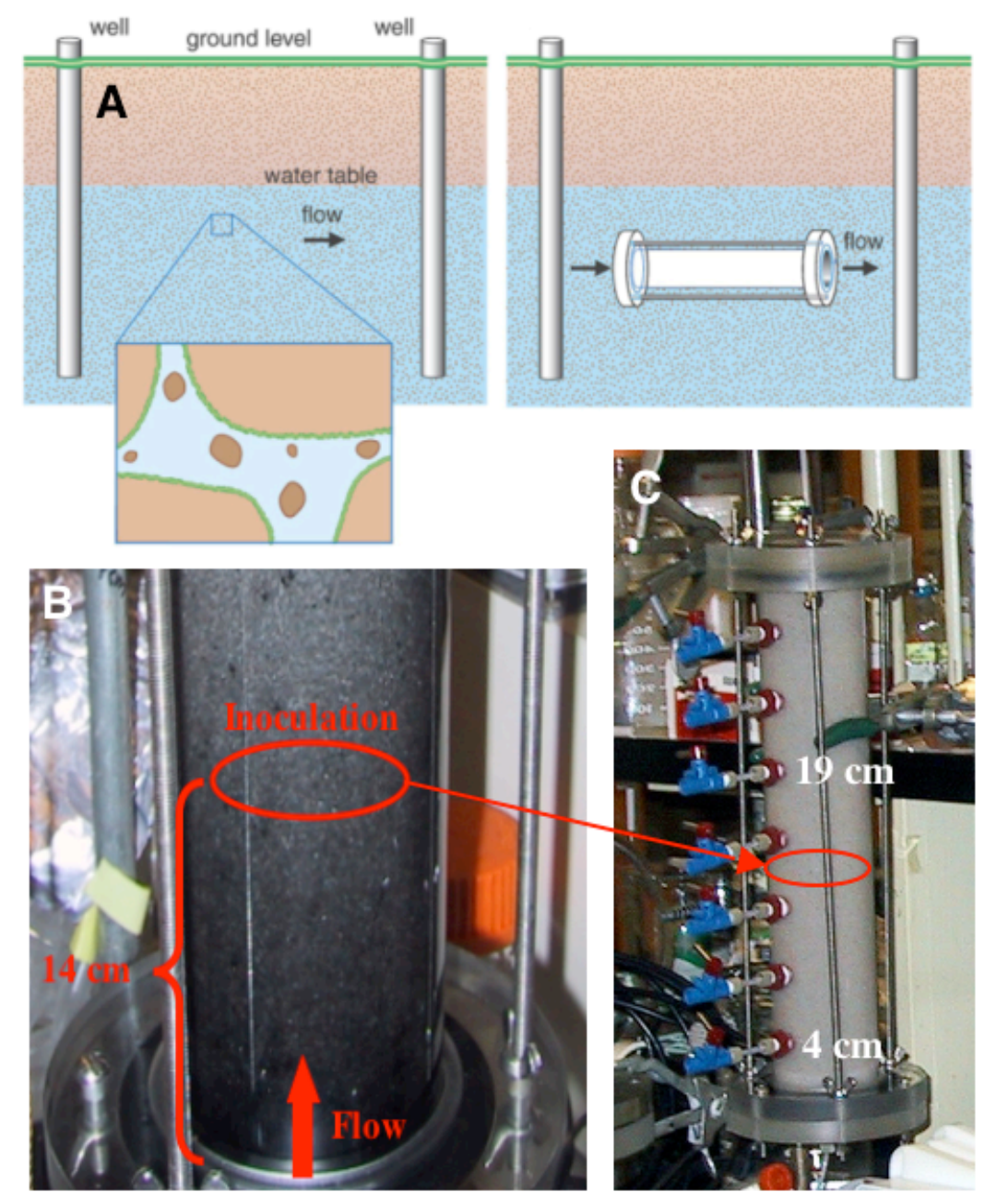

Figure S1 
Figure S1. (A) Schematic representation of an aquifer model, pore-scale biomineralization, and column apparatus. (B) Photograph of the acoustic wave column on day 20 showing the flow direction, the location of initial inoculation, and dark-colored sulfide mineralization near the base. (C) Photograph of the multi-port fluid sampling column showing the location of the 4 and $19 \mathrm{~cm}$ positions referred to in the text.



Figure S2

Figure S2. Photograph of the column used to make complex resistivity measurements illustrating the 
electrode design and layout. The inset image highlights the changes associated with biomineralization after 31 days in the region $4 \mathrm{~cm}$ from the influent end of the column.

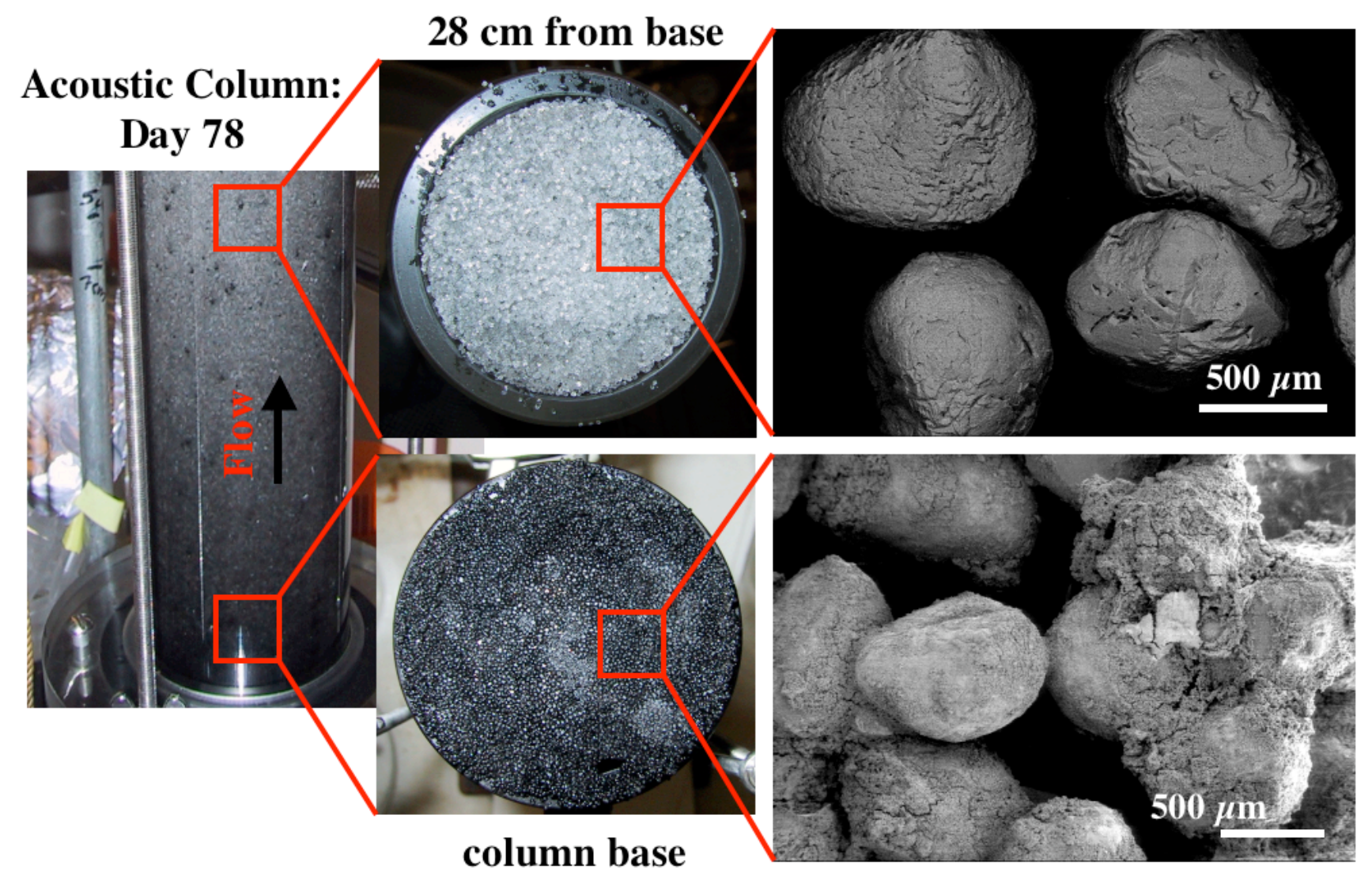

Figure S3

Figure S3. Photograph of the column used to make acoustic wave measurements illustrating the changes in sediment characteristics within the same column after 78 days at locations near the influent end (i.e. the column base) and $28 \mathrm{~cm}$ downgradient. The SEM images of sand grains from the $28 \mathrm{~cm}$ location are similar in character to those extracted from the non-inoculated controls. Extensive alteration of the pore throat openings as a result of mineral precipitation is evident in the sand grains extracted from the location nearest the influent end. 

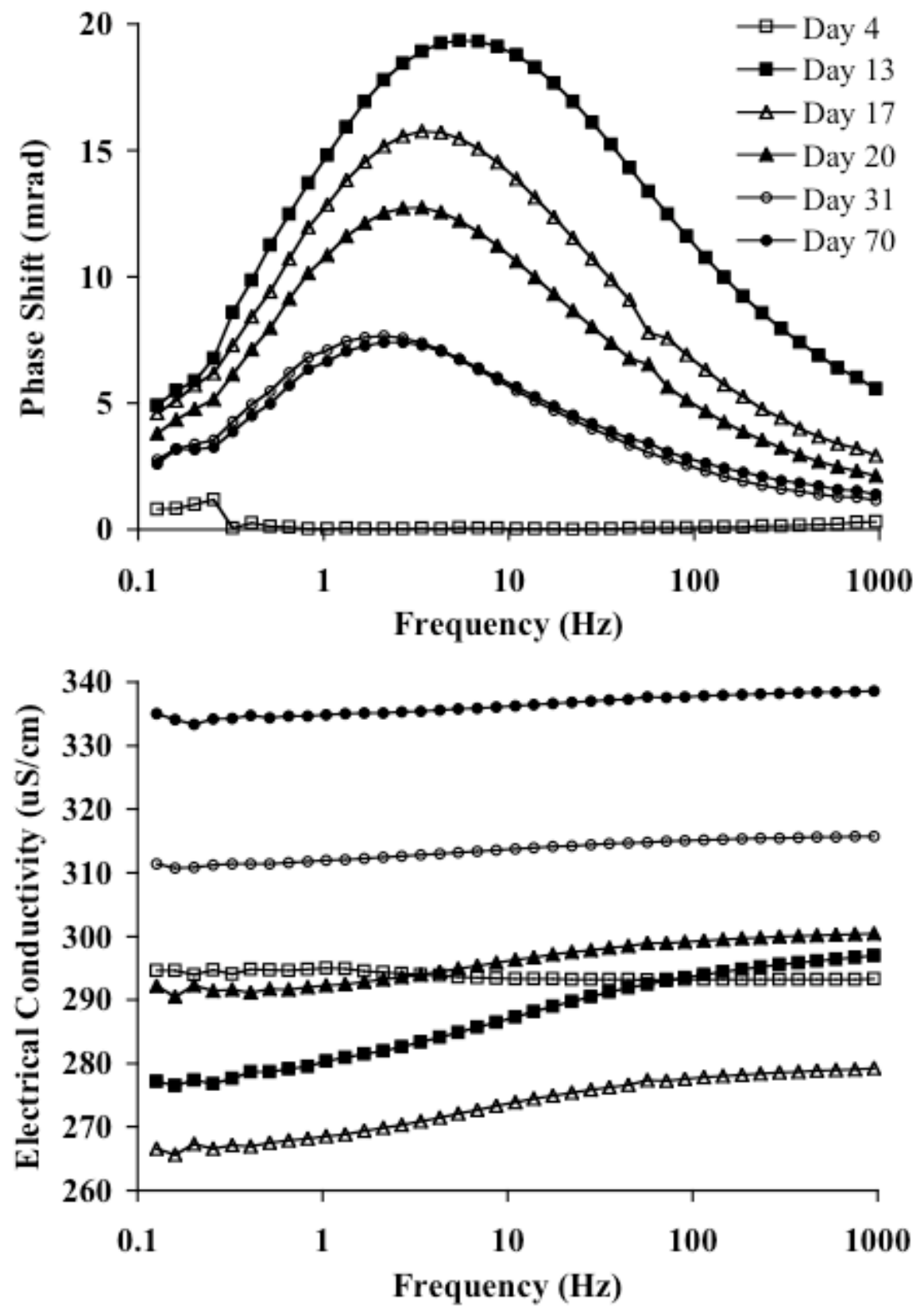

Figure S4

Figure S4. Temporal development of the $4 \mathrm{~cm}$ phase anomaly showing the relationship between bulk electrical conductivity and phase response; legends are identical for both images. Decreases in the phase response inversely track increases in bulk electrical conductivity. This observation supports the hypothesis that growth and aging of FeS nanocrystals led to the creation of electrically conductive shortcircuits along grain surfaces that acted to diminish the phase response with time. 